\title{
Peripartum cardiomyopathy: when labour turns to heartbreak
}

\author{
Edgar LW $\underline{T \text { ayy }}{ }^{1,2}$, MRCP, FACC, James WL $\underline{\text { Yip }}^{1,2}$, MBBS, MRCP, Kian Keong $\underline{P o h^{1,2}}$, FRCP, FACC
}

T he association between heart failure and pregnancy was described in 1850 by Ritchie, ${ }^{(1)}$ but it was first described as a distinct clinical pathological entity by Gouley in 1937.(2) In 1971, the term 'peripartum cardiomyopathy' (PPCM) was coined by Demakis, ${ }^{(3)}$ who defined the diagnostic criteria that was later adapted for use by the National Heart Lung and Blood Institute (NHLBI). The four criteria for defining PPCM recommended by the NHLBI include: (a) the development of cardiac failure in the last month of pregnancy or within five months of delivery; (b) the absence of an identifiable cause of cardiac failure; (c) the absence of a recognisable heart disease prior to the last month of pregnancy; and (d) left ventricular systolic dysfunction demonstrated by echocardiographic criteria such as depressed left ventricular ejection fraction. ${ }^{(4)}$ Although the aetiology of PPCM is unclear, a recent study has suggested that antiangiogenic factors produced at the final stage of pregnancy may be related to this condition. ${ }^{(5)}$

The treatment of PPCM is similar to that of other types of heart failure, with the use of vasodilators, nitrates and diuretics being the mainstay of therapy in acutely ill or symptomatic patients. Although the use of angiotensin-converting enzyme inhibitors is contraindicated during pregnancy due to the risk of foetal teratogenicity, they are the mainstay of therapy after delivery, even in mothers who are breastfeeding. ${ }^{(6)}$ Beta-blocker usage during pregnancy is associated with low birthweight babies, but if required, metoprolol is the preferred choice. Thromboembolism, which is a common occurrence in this population, may be treated with subcutaneous heparin (5,000 units twice daily). ${ }^{(7)}$ Therefore, therapeutic decisions are often influenced by the drug-safety profiles during pregnancy and lactation. In addition, mechanical support and transplantation may be necessary in severe cases. Novel and targeted therapies such as immunotherapy, however, remain to be evaluated.

The true incidence of PPCM worldwide is still unknown. The accepted incidence is one per 3,000-4,000 births, although it is known to be rare in Europe, ${ }^{(8)}$ and more common in West Africa ${ }^{(9)}$ and Haiti. ${ }^{(10)}$ The Hausa tribe in Nigeria has been reported to have the highest incidence, with $13 \%$ of all female admissions found to have this condition. ${ }^{(11)}$ The reported incidence of PPCM varies widely, from 1:100 to 1:15,000 live births, and this could be attributed to the differing definitions of PPCM and referral patterns in different countries. ${ }^{(3)}$ In largely western cohorts, a more likely estimate is 10-20 per 100,000 live births. ${ }^{(4)}$

In this issue of the $S M$ J, we highlight two articles - one by Lim and $\operatorname{Sim}^{(12)}$ (page 24), and the other by $\operatorname{Chee}^{(13)}$ (page 28), which have made important contributions to the field of PPCM in Asia. To date, the two series are the largest cohorts characterising this condition among Asians. In the series by Lim et al, the incidence of PPCM was found to be 0.89 per 1,000 births or one per 1,123 births, while the prevalence over a period of ten years in Chee's series was even lower, at 2.48 per 100,000 live births. This distinct difference in incidence between the two studies suggests a high possibility of referral bias, as well as the inherent difficulty in distinguishing patients with PPCM from those who may have pre-existing asymptomatic dilated cardiomyopathy prior to pregnancy. Novel imaging parameter or serum biomarker may help to differentiate this in the future. ${ }^{(14)}$

The traditional risk factors for PPCM include multiparity, hypertension, advanced maternal age, black race, the use of cocaine and tocolytic therapies. Although there is an overlap of risk factors in these two studies, the small number of patients in both series makes it difficult to draw clear conclusions. In Lim et al's series, Malay patients were found to have a higher incidence of PPCM when adjusted for racial birth rates, ${ }^{(12)}$ while $50 \%$ of the cohort was Malay in Chee's study. ${ }^{(13)}$ Here again, the numbers are too small to draw any firm conclusions.

There was one maternal death and two (17\%) patients requiring mechanical ventilation in Chee's series, ${ }^{(12)}$ whereas no deaths and four (36\%) patients needing mechanical ventilation were noted in Lim et al's study. ${ }^{(13)}$ From the available information in the two articles, it appears that approximately $60 \%$ of the patients recovered. The recovery rates, according to the literature, vary from $23 \%-65 \%,{ }^{(15,16)}$ with a reported recovery time of about six months, although this is also variable.(17,18) Patients with severe left ventricular dilatation and severe reduction of left ventricular ejection fraction at presentation were much less likely to recover. A recent study on 176 African women with newly diagnosed PPCM suggests that at baseline, increased left ventricular endsystolic dimension (LVESD) on echocardiography, a lower body mass index and a lower serum cholesterol level may be predictors of poor outcome in patients with PPCM, while older age and smaller LVESD at baseline appear to be associated with a higher chance of left ventricular recovery. ${ }^{(19)}$

\footnotetext{
${ }^{1}$ Cardiac Department, National University Heart Centre, National University Health System, ${ }^{2}$ Department of Medicine, Yong Loo Lin School of Medicine, Nationa University of Singapore, Singapore

Correspondence: A/Prof Poh Kian Keong, Senior Consultant and Associate Professor, Cardiac Department, National University Heart Centre, 1E Kent Ridge Road, NUHS Tower Block, Level 9, Singapore 119228. kian_keong_poh@nuhs.edu.sg
} 
The recurrence rates of PPCM reported in the existing literature vary from $25 \%-100 \%{ }^{(20)}$ The two studies were likewise unable to present any indication of the recurrence rate, given the small number of patients in the series. Importantly, patients with impaired left ventricular ejection fraction should be counselled against another pregnancy, as the risks of mortality (19\%) and of developing heart failure (up to $40 \%$ ) may be too high a price to pay. Advice for patients with restored left ventricular function who wish to undertake pregnancy is less certain. The risks in this group include a significant fall in left ventricular function (up to $20 \%$ ) and permanent left ventricular dysfunction (up to $21 \%$ ). ${ }^{(21)}$

In summary, both Chee and Lim et al have shown in their series that PPCM is an uncommon condition that can result in significant maternal morbidity. The authors have also demonstrated that approximately $60 \%$ of patients show recovery of their left ventricular ejection fraction over time. The two papers have raised important questions, which we hope will spur more research in this field, and have also illustrated the need for multicentre systematic collaborative research in order to address rare but important diseases.

\section{REFERENCES}

1. Ritchie $\mathrm{C}$. Clinical contribution to the patho-diagnosis and treatment of certain chronic diseases of the heart. Edinb Med J 1850; 2:2.

2. Gouley BA, McMillan TM, Bellet S. Idiopathic myocardial degeneration associated with pregnancy and especially the puerperium. Am J Med Sci 1937; 19:185-99.

3. Demakis JG, Rahimtoola SH, Sutton GC, et al. Natural course of peripartum cardiomyopathy. Circulation 1971; 44:1053-61.

4. Pearson G, Veille J, Rahimtoola S, et al. Peripartum cardiomyopathy: National Heart, Lung and Blood Institute and Office of Rare Diseases (National Institutes of Health) workshop recommendations and review. JAMA 2000; 283:1183-8.

5. Patten IS, Rana S, Shahul S, et al. Cardiac angiogenic imbalance leads to peripartum cardiomyopathy. Nature 2012; 485:333-8.

6. Mastrobattisha JM. Angiotensin converting enzyme inhibitors in pregnancy. Semin Perinatol 1997; 21:124-34.
7. Abboud J, Murad Y, Chen-Scarabelli C, Saravolatz L, Scarabelli TM. Peripartum cardiomyopathy: a comprehensive review. Int J Cardiol 2007; 118:295-303.

8. Fett JD, Dowell DL, Carraway RD, Sundstrom JB, Ansari AA. One hundred cases of peripartum cardiomyopathy... and counting: what is going on? Int J Cardiol 2004; 97:571-3.

9. Ferriere M, Sacrez A, Bouhour JB, et al. [Cardiomyopathy in the peripartum period: current aspects. A multicenter study. 11 cases]. Arch Mal Coeur Vaiss 1990; 83:1563-9. French.

10. Cenac A, Djibo A. Postpartum cardiac failure in Sudanese-Sahelian Africa: clinical prevalence in western Niger. Am J Trop Med Hyg 1998; 58:319-23.

11. Davidson NM, Parry EH. Peri-partum cardiac failure. Q J Med 1978; 47:431-61.

12. Lim CP, Sim KL. Peripartum cardiomyopathy: experience in an Asian tertiary centre. Singapore Med J 2013; 54:24-7.

13. Chee KH. Favourable outcome after peripartum cardiomyopathy: a ten-year study on peripartum cardiomyopathy in a university hospital. Singapore Med J 2013; 54:28-31.

14. Walenta K, Schwarz V, Schirmer SH, et al. Circulating microparticles as indicators of peripartum cardiomyopathy. Eur Heart J 2012; 33:1469-79.

15. Lampert MB, Weinert L, Hibbard J, et al. Contractile reserve in patients with peripartum cardiomyopathy and recovered left ventricular function. Am J Obstet Gynecol 1997; 176 (1 Pt 1):189-95.

16. Elkayam U. Pregnant again after peripartum cardiomyopathy: to be or not to be? Eur Heart J. 2002; 23:753-6.

17. Biteker M, Ilhan E, Biteker G, Duman D, Bozkurt B. Delayed recovery in peripartum cardiomyopathy: an indication for long-term follow-up and sustained therapy. Eur J Heart Fail 2012; 14:895-901.

18. Mouquet F, Mostefa Kara M, Lamblin N, et al. Unexpected and rapid recovery of left ventricular function in patients with peripartum cardiomyopathy: impact of cardiac resynchronization therapy. Eur J Heart Fail 2012; 14:526-9.

19. Blauwet LA, Libhaber E, Forster O, et al. Predictors of outcome in 176 South African patients with peripartum cardiomyopathy. Heart 2012 October 31 [Epub ahead of print].

20. Hibbard JU, Lindheimer M, Lang RM. A modified definition for peripartum cardiomyopathy and prognosis based on echocardiography. Obstet Gynecol 1999; 94:311-6.

21. Elkayam U, Tummala PP, Rao K, et al. Maternal and fetal outcomes of subsequent pregnancies in women with peripartum cardiomyopathy. $\mathrm{N}$ Engl J Med 2001; 344:1567-71. Erratum in: N Engl J Med 2001; 345:552. 